Zornberg J. G., Costa, Y.D., and Bueno B.S. (2005). "Failure Mechanisms in Pipelines Bridging a Void.” Geosynthetics Research and Development in Progress, Eighteenth Geosynthetic Research Institute Conference (GRI-18), Austin, Texas, January 26 (CD-ROM).

\title{
Failure Mechanisms in Pipelines Bridging a Void
}

\author{
J. G. Zornberg ${ }^{1}$, Y.D. Costa ${ }^{2}$, and B.S. Bueno ${ }^{3}$ \\ ${ }^{1}$ Civil Engineering Department, University of Texas at Austin, Austin, TX 78712; \\ PH (512) 232-3595; FAX (512) 471-6548; email: zornberg@mail.utexas.edu \\ ${ }^{2}$ Department of Geotechnical Engineering, University of Sao Paulo, Sao Carlos, \\ Brazil; email: ydjcosta@sc.usp.br \\ ${ }^{3}$ Department of Geotechnical Engineering, University of Sao Paulo, Sao Carlos, \\ Brazil; email: bsbueno@sc.usp.br
}

\section{Abstract}

An experimental testing program is being conducted to evaluate the failure mechanisms of pipelines subjected to a localized loss of support. The program involves a series of centrifuge tests conducted to evaluate the response of pipes embedded in sand backfill and placed over a rigid trapdoor base that is released during testing. The tests involve a pipe with prototype diameter of $1.1 \mathrm{~m}$ and a soil cover height of approximately 5 times the pipe diameter. Failure patterns are observed along longitudinal and transverse cross-sections of the pipe-soil system. The influence of pipe stiffness and backfill density on the behavior of the system is preliminary assessed in this paper. The transverse sections showed the development of well-defined slip surfaces initiating at the edge of the void. Slip surfaces did not develop along the longitudinal cross sections. The mode of failure of flexible pipes involved buckling in the shoulder of the pipe and a reversal of curvature at the invert due to excessive deflection. Preliminary test results indicate that the density of the surrounding soil has a significant effect on the damage experienced by flexible pipes.

\section{Introduction}

This paper presents preliminary results of an experimental study aimed at identifying the failure mechanisms of pipes subjected to loss of support. An important outcome is that proper identification of the failure mechanisms will allow the design of reinforced soil systems suitable to mitigate damage in pipelines. The experimental program includes a series of centrifuge tests involving tubes buried in dry sand backfill. The tubes rest over a rigid base (trapdoor) that moves downwards during testing. Failure patterns were documented within a vertical longitudinal section along the axis of the pipe as well as in four transverse vertical sections at varying distances from the trapdoor. The different tests were conducted to evaluate the effect of the pipe stiffness and backfill density on the response of pipes bridging a void. 


\section{Centrifuge Tests}

The reduced-scale models were built using half-pipes because of the longitudinal symmetry of the problem, which allowed visualization of the failure mechanism along the axis of the pipe. The rigid box used to contain the models was $419 \mathrm{~mm}$ long, $203 \mathrm{~mm}$ wide and $300 \mathrm{~mm}$ high. The front wall of the rigid box is a transparent Plaxiglass plate that enables in-flight visualization of the models during testing. The trapdoor base, which was located adjacent to the transparent wall, could be lowered during in-flight testing using an electromagnetic release system. Fig. 1(a) shows the vertical cross-section of one of the models indicating the location of the moving base (trapdoor), half-pipe, and colored sand layers placed to document the displacements within the backfill soil. Figure 1(b) shows the plan view of a typical model, and indicates the location of the half-pipe and rigid trapdoor base (against the transparent wall) as well as the location of the four cross-sections (S1 to S4) that are dissected after testing for evaluation of the failure mechanisms.
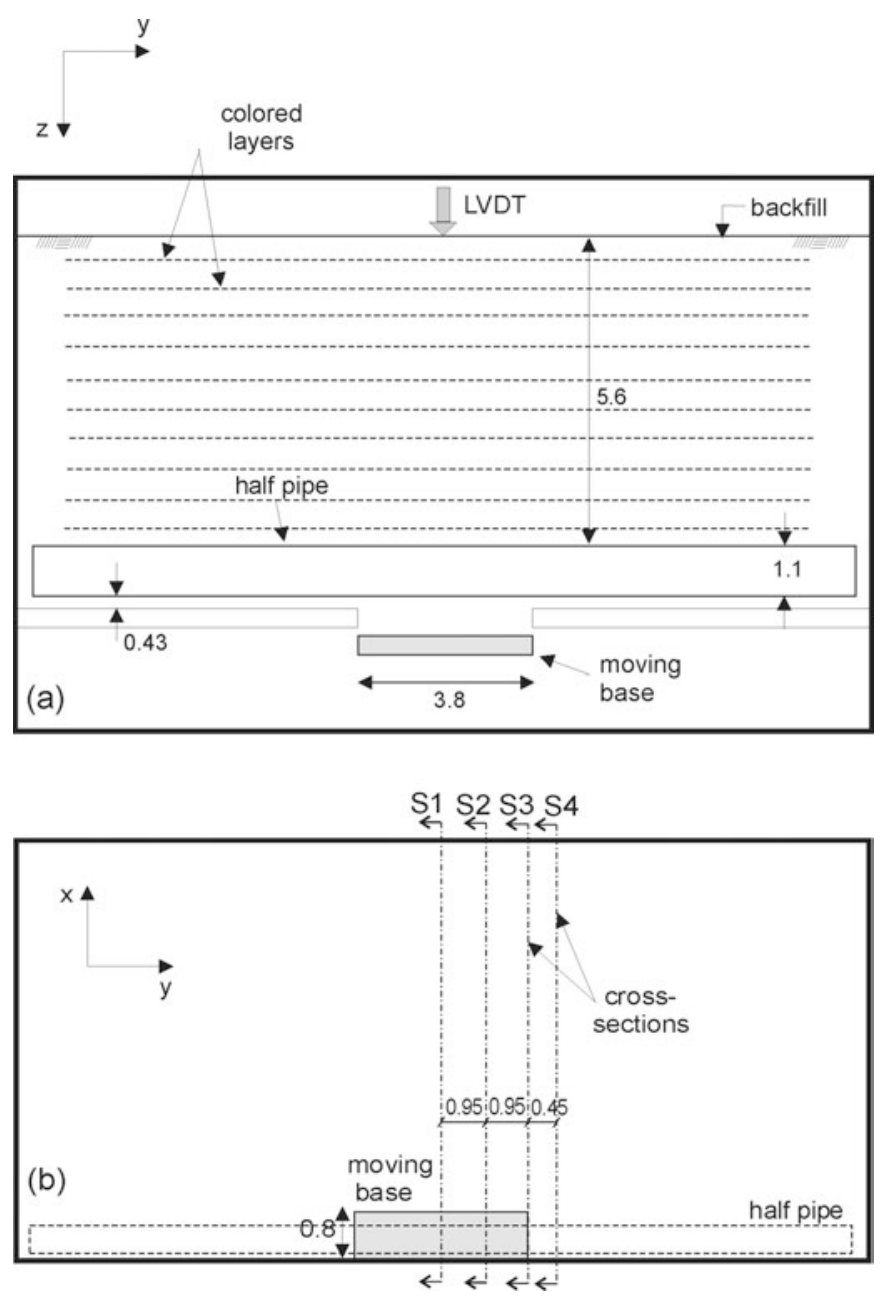

Figure 1. Layout of the centrifuge tests (note: dimensions in the figure are prototype values in $(\mathbf{m})$. 
The pipe models used in this study consisted of half-section tubes with smooth surface. The pipes had an outside diameter $(D)$ of $25.4 \mathrm{~mm}$, a length $(L)$ of $400 \mathrm{~mm}$, and a wall thickness $(t)$ of either 0.127 or $0.7 \mathrm{~mm}$. The selected wall thickness values were adopted to represent flexible (F) and rigid (R) pipes, respectively.

The centrifuge tests were performed in using a 15 g-ton centrifuge with a nominal radius of $1.36 \mathrm{~m}$, and with capability of accelerating a $135-\mathrm{kg}$ payload to $100 \mathrm{~g}$. All models were tested under a nominal centrifuge acceleration of $45 \mathrm{~g}$.

All models were prepared using dry Ottawa F-75 sand, which is a fine, uniformly graded silica sand with an average particle size of $0.22 \mathrm{~mm}$. The models were prepared by pluviating air-dried sand under controlled conditions into the assembled testing apparatus. A relative density of $42 \%$ was selected to represent a loose backfill, while a relative density of $85 \%$ was chosen to represent a dense backfill. The target relative densities $\left(D_{r}\right)$ of 85 and $42 \%$ were achieved by pluviating the sand from specific elevations and using pre-defined discharge rates. The angle of internal friction $(\phi)$ of the sand, obtained from plane strain tests, is 40 and $48^{\circ}$ for $D_{\mathrm{r}}$ of 42 and $85 \%$, respectively.

A soil cover with a prototype height $(\mathrm{H})$ of $5.6 \mathrm{~m}$ was used in the models, which correspond to a cover depth ratio $H / D$ of approximately 5. Installations are considered to correspond to deep burial conditions when the cover depth ratio $H / D$ exceeds approximately 2.5 (Sloan et al. 1990). The electromagnetic system holding the rigid trapdoor base in place was released during centrifuge testing. This induced a vertical movement of $20 \mathrm{~mm}$, which corresponds to a vertical movement in prototype dimensions of $0.9 \mathrm{~m}$. The failure mechanisms were evaluated based on inspection of ten thin horizontal layers of colored sand placed within the soil at vertical intervals of $12.7 \mathrm{~mm}$ (Fig. 1a). The displacements of the colored layers, both before and after lowering the rigid trapdoor base, were photographically recorded in-flight through the transparent wall of the strongbox. In addition, the models were dissected after testing by wetting the sand model. This allowed evaluation of the failure patterns along the transverse sections S1 to S4 (Fig. 1b). These sections were located at increasing distances from the center of the rigid trapdoor base. Finally, the settlement at the backfill surface of the backfill $\left(\delta_{\mathrm{s}}\right)$ was recorded using an LVDT positioned directly above the center of the rigid trapdoor base.

Four centrifuge tests were conducted as part of this investigation. The main characteristics of the tests are summarized in Table 2. Only the results corresponding to Model 1 (flexible pipe in loose soil) are presented in this paper.

\section{Preliminary Results}

Figure 2 shows the failure patterns observed in sections S1, S2 and S3 of Model 1 (flexible pipe in loose soil). Well-defined slip surfaces developed within the soil mass, which are represented by dashed lines in the figure. The failure pattern involved three distinct slip surfaces that propagated from edge of the void. The innermost of the three slip surfaces developed by sliding of soil adjacent to the pipe into the underlying void. Instead, the other two slip surfaces appear to have 
developed from subsequent instability of the soil mass next to the moving base and by the deformations of the pipe itself.

\begin{tabular}{lllll}
\multicolumn{5}{c}{ Table 1. Test Characteristics } \\
\hline Test & $\begin{array}{l}\mathrm{D}_{\mathrm{r}} \\
(\%)\end{array}$ & $\begin{array}{c}\text { Pipe } \\
\text { type }\end{array}$ & $\begin{array}{l}\text { g- } \\
\text { level }\end{array}$ & $\begin{array}{l}\boldsymbol{\delta} \\
(\mathrm{m})\end{array}$ \\
\hline 1 & 42 & $\mathrm{~F}$ & 45 & 0.9 \\
2 & 85 & $\mathrm{~F}$ & 45 & 0.9 \\
3 & 42 & $\mathrm{R}$ & 45 & 0.9 \\
4 & 85 & $\mathrm{R}$ & 45 & 0.9 \\
\hline
\end{tabular}
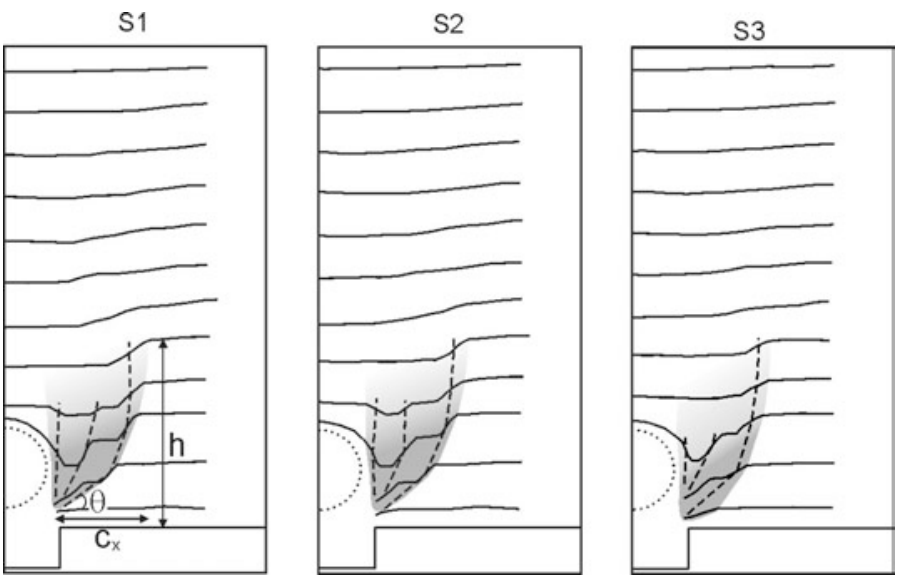

Figure 2. Failure pattern observed in the transverse sections of Model 1 (flexible pipe in loose backfill).

Inspection of the failure patterns indicates that the localized loss of support experienced by the flexible pipe in the loose backfill caused a failure zone with a smaller height $(h)$ and horizontal extent $\left(c_{\mathrm{x}}\right)$ than in the rigid pipe embedded in loose backfill. Also, the effect of the pipe stiffness on the surficial settlement was found to be negligible, as measured by the readings of the LVDT located directly above the rigid trapdoor base.

Unlike the transverse cross-sections, well-defined slip surfaces did not develop in the longitudinal cross-sections of the models. Instead, zones of subsidence were observed in the soil mass due to the migration of the material into the underlying void. Figure 3 shows the longitudinal failure patterns in Model 1. As in the transverse sections, the extent of the failure zone was quantified by the length of its projection in the $x-y$ plane $\left(c_{\mathrm{y}}\right)$ measured from the edge of the void. The extent of the failure zone is smaller for the rigid pipe than for the flexible pipe when using loose backfill. Also, the extent of the failure zone is negligible in the dense backfill, regardless the pipe stiffness. Small cavities developed in the vicinity of the edges of the void, as indicated in Figure 3. Evaluation of the extent of the failure zone is useful for the validation of simplified analytical methods such as those based on the determination of the modulus of subgrade reaction (Vesic 1961).

In addition to assessment of the failure pattern within the soil mass, the failure mechanism of the pipe itself was also evaluated in this study. For example, failure of the flexible pipe embedded in the loose backfill (Model 1) involved buckling in the shoulder and crown of the pipe, as well as a reversal of curvature at the invert due to excessive deflection. Figure 4 shows the damage in the pipe (Model 1) after testing. The arrows indicate the damage in the shoulder of the pipe along the transverse crosssection $\mathrm{S} 1$. As observed in this transverse cross-section, the vertical deflection $(\Delta / D)$ of the pipe induced by the movement of the underlying base was approximately $26 \%$. Preliminary test results indicate that the density of the surrounding soil has a 
significant effect on the damage experienced by flexible pipes.

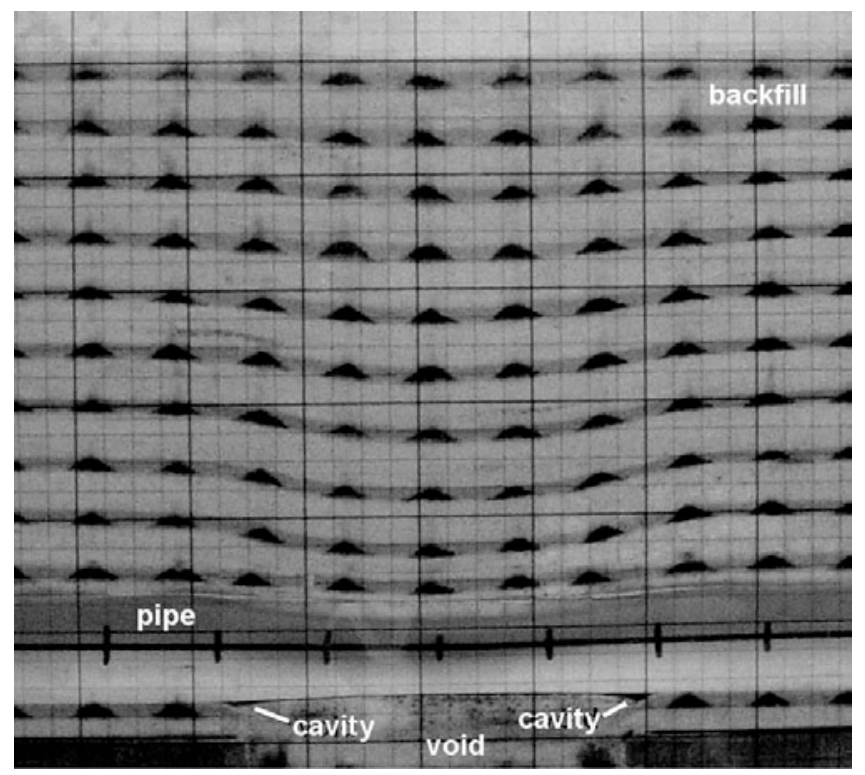

Figure 3. Failure pattern observed in the longitudinal section of Model 1 (flexible pipe in loose backfill).

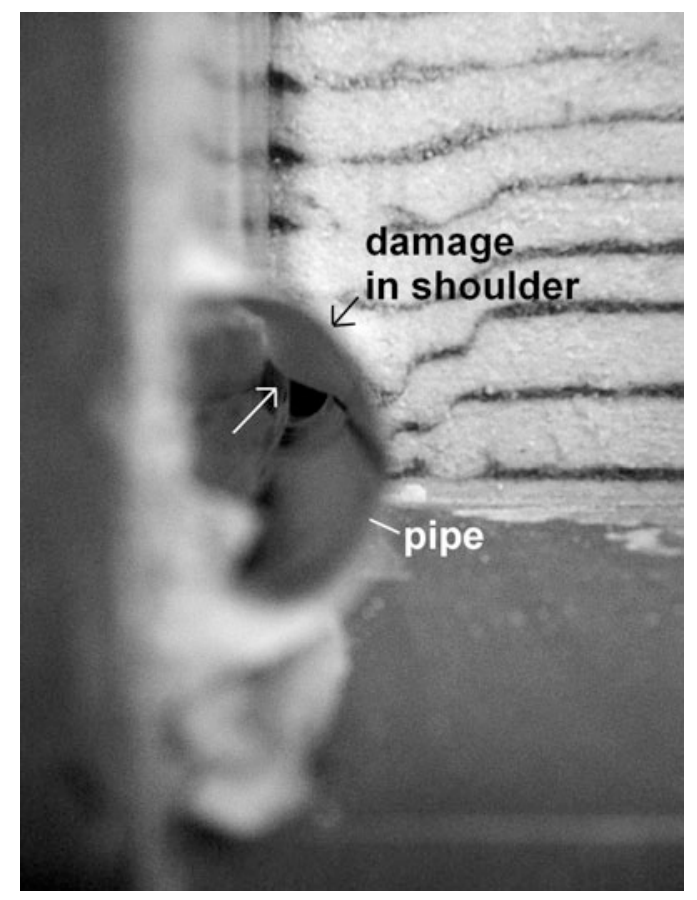

Figure 4. Damage experienced by the flexible pipe in Model 1 (Section S1). 


\section{Preliminary Conclusions and Path Forward}

A series of centrifuge tests was conducted to investigate the response of pipelines subjected to ground loss under deep burial conditions. The failure mechanisms were investigated by analysis of the longitudinal and transverse sections within the soil mass. Analysis of the transverse sections revealed well-defined slip surfaces propagating from edge of the void. Rigid pipes caused comparatively larger failure zones in both loose and dense backfills. The magnitude of the surficial settlement showed to be independent of the pipe stiffness. Flexible pipes embedded in loose backfill experienced buckling in the shoulder and crown of the pipe, and a reversal of curvature in the invert due to excessive deflection.

Continued efforts in this investigation include the development of analytical solutions for calculating settlements of buried pipelines. Also, design methods for geosynthetic reinforced soil, which are consistent with the failure mechanisms observed in this study, are being developed for projects involving pipelines subject to ground loss.

\section{References}

Sloan, S. W., Assadi, A., Purushothaman, N., 1990, "Undrained Stability of a Trapdoor," Géotechnique, vol. 40, no. 1, pp. 45-62.

Vesic, A. S., 1961, "Bending of Beams Resting on Isotropic Elastic Solid," Journal of Engineering Mechanics Division, ASCE, vol. 87, no. 2, pp. 35-53. 